\title{
Catálogo de tipos depositados en el herbario del Museo Argenti- no de Ciencias Naturales "Bernardino Rivadavia" (BA). II. Monocotiledóneas: Poaceae
}

\author{
Mirta O. ARRIAGA", Roberto A. GÓMEZ CADRET² \& Ma. Dolores MONTERO² \\ Laboratorio de Anatomía Vegetal y Herbario de Plantas Vasculares, Museo Argentino de Ciencias Naturales \\ "Bernardino Rivadavia". Av. Angel Gallardo 470, C1405DJR Buenos Aires, Argentina. ${ }^{3}$ Carrera Investigador \\ Científico, CONICET. ${ }^{2}$ Carrera Personal de Apoyo a la Investigación, CONICErT.
}

\begin{abstract}
The herbarium of the Argentinian Musetum of Natural Sciences (BA Herbarizm) was the first official therbarium in this country. It was initiated in 1853 and at present holds 150,000 herbarium samples from different points of Argentina. From them about 100,000 correspond to vascular plants and 800 represent nomenclatuxal type specimens. The present contribution is the second part of the "BA Alerbarium type specimens catalog" and presents information on 112 type specimens of the family Poaceae. The specimens are alphabetically ordered, and species name, author, original citation and date, type classification, and all label data are transcribed. Under observations, ecological data or additional notes written by the author of the description are given. Under notes, some considerations of our own, or the current synonymy are given. Type specimens are kept in special metal cabinets, separated from the general herbarium and documented with a copy of the original diagnosis and illustration. A database was developed with the program ACCESS 2000.
\end{abstract}

Key words: BA herbarium, Monocots, Poaceae, type specimens, Argentina.

E1 Herbario del Museo Argentino de Ciencias Naturales "Bernardino Rivadavia", herbario BA (Holmgren et al., 1990) constituye la Colección Nacional de Botánica, iniciada en 1853 como parte del entonces Museo Nacional de Historia Natural, representando el primer herbario oficial de nuestro país. En la actualidad alberga unos 150.000 ejemplares coleccionados en distintos puntos de nuestro territorio, enriquecido también con materiales obtenidos sobre la base de canje con distintas instituciones nacionales o extranjeras. De esta totalidad de especímenes alrededor de 100.000 corresponden al grupo de Plantas Vasculares, entre los que se han detectado unos 800 ejemplares tipo.

La presente constituye la continuación del Catálogo de tipos depositados en el Herbario BA, cuya primera parte presenta 30 especimenes tipo pertenecientes a las familias Potamogetonaceae, Alismataceae, Cyperaceae, Xyridaceae y Eriocaulaceae (López, 1988). En esta oportunidad se dan a conocer 112 especímenes tipo pertenecientes a la familia Poaceae.

\section{DATOS DEL FORMATO}

Los especímenes fueron ordenados alfabéticamente, citándose el nombre de la especie, autor, y obra original. Luego se agrega la clasificación del tipo de nomenclatura correspondiente a cada ejemplar (según Código 1983). Se transcribe a continuación, exactamente los datos que figuran en la etiqueta original: a) lugar de colección (país, provincia, departamento, localidad); b) legit (colector; $\mathrm{n}^{\circ}$ de colector, fecha de colección consignada como día/mes/año); BA no (número de ingreso del ejemplar a la colección de este herbario). Algunos de estos datos pueden faltar originalmente. En observaciones figuran otros datos de la etiqueta de herbario (altitud, hábito de la planta, hábitat, etc), datos de la etiqueta de campo, o anotaciones realizadas por el autor de la descripción.

En algunos casos figura una nota (a veces más de una), realizada por los autores del presente catálogo, donde se consigna aclaraciones o datos de la actual sinonimia para cada nombre. 
Cada uno de los ejemplares fue documentado, con una copia de la diagnosis original, adjunta en la carpeta de herbario (Fig. 1).

Se realizó una base de datos, desarrollada con ACCESS 2000 de Microsoft, mediante la cual puede accederse a la información desde distintos campos.

\section{CATALOGO}

Agropyron anderetedem Parodi. Parodi, L.R. 1940. Estudio crítico de las Gramineas AustralAmericanas del género Agropyron. Rev. Mus. La Plata III, Bot. 12: 48-51, fig. 17-18.

Holotipo: Chile: Puerto Soffia, Isla Barrow (archipiélago fueguino). Leg. A. Castellanos. Fecha: 03/1934. BA 21929.

Nota: un estudio posterior (Seberg, 1989), sinonimiza esta especie con Elymus glaucescens Seberg.

Agropyrow athertacen (H.B.K.) Roem. et Schult. var. arcucanum Parodi. Parodi, L.R. 1940. Estudio crítico de las Gramineas AustralAmericanas del género Agropyron. Rev. Mus. La Plata III, Bot. 12: 35-36, fig, 12, Larn. VIII.

Holotipo: Argentina: Neuquén, Cordillera del Viento. Leg. A. Ragonese 94. Fecha: 16/01/1935. BA 14331.

Nota 1: un estudio posterior (A. Löve, 1984), sinonimiza esta variedad con Elymus araucanus (Parodi) A. Löve. Nota 2: en la descripción original figura el ejemplar como Buenmus n²14331 en lugar de BA 14331.

Agropyron attenctum (H.B.K.) Roem. et Schult. var. ruizianum Parodi, Parodi, L.R. 1940. Estudio crítico de las Gramineas AustralAmericanas del género Agropyron. Rev. Mus. La Plata III, Bot. 12: 34.

Isotipa: Argentina: Mendoza, Crucesita (Precordillera). Leg. E. Carette (Herb. L. Hauman). Fecha: 12/1906. BA 40550.

Nota: según Zuloaga et al. (1994) constituye un taxón dudoso, "posiblemente sinónimo de Elymus glaucescens Seberg (Seberg, 1989)".

Agropyron fuegianum (Speg.) Kurtz var. brachyatherum Parodi. Parodi, L.R. 1940. Estudio critico de las Gramíneas Austral- Americanas del género Agropyron. Rev. Mus. La Plata III, Bot. 12: 55-57, fig. 20, Lam. XII.

Paratipo: Argentina: Isla Grande de Tierra del Fuego, prope Río Grande, Segunda Argentina. Leg. A. Castellanos. Fecha: 13/01/1933. BA $7600 \%$.

Nota 1: un estudio posterior (Seberg, 1989) sinonimiza esta variedad con Elymus glaucescens (Seberg, 1989). Nota 2: en la descripción original figura el ejemplar como Castellanos $n^{\circ} 7600 \mathrm{~L} / 2$ (Buenmus) en lugar de Castellanos s/n, BA 76001/2.
Agropyron fuegianum (Speg.) Kurtz var. chaetophorum Parodi. Parodi, L.R. 1940. Estudio crítico de las Gramíneas Austral- Americanas del género Agropyron. Rev. Mus. La Plata III, Bot. 12: 57-59, fig. 21.

Holotipo: Argentina: Isla Grande de Tierra del Fuego, prope Río Grande, Segunda Argentina. Leg. A. Castellanos. Fecha: 13/01/1933. BA 7600. Nota: según Zuloaga et al. (1994) constituye un taxón dudoso.

Agropyron fuegianum (Speg.) Kurtz var. chaetophorum Parodi. Parodi, L.R. 1940. Estudio crítico de las Gramíneas Austral- Americanas del género Agropyron. Rev. Mus. La Plata III, Bot. 12: 57-59.

Paratipo: Argentina: Tierra del Fuego, Arroyo Chapel, Estancia «Tepi». Leg. A. Castellanos. Fecha: 22/01/1933. BA 7613 .

Nota 1: según Zuloaga et al. (1994) constiztuye un taxón dudoso.

Nota 2: en la descripción original figura el ejemplar como Castellanos no7613 (Buenmus) en lugar de Castellanos s/n, BA 7613 .

Agropyron magellanicum (Desv.) Hack. var. glabpivalva (Speg.) Hauman et Vanderveken. Spegazzini, C. 1896. Plantae per Fuegiam a Carolo Spegazzini, anno 1882, Collectae. An. Mus. Nac. Buenos Aires 5: 98. Hauman, L. et G. Vanderveken. 1917. Catalogue des Phanèrogames de l'Argentine. An. Mus. Nac. Hist. Nat. Buenos Aires 29: 1-351.

Paratipo: Argentina; "Tierra del Fuego». Leg. Spegazzini: Pl. Fuegiam coll. 286. Fecha: 1882. BA 40555.

Nota: un estudio posterior (Seberg, 1989) sinonimiza esta variedad con Elymus glaucescens

Agropyron patagonicum (Speg.) Parodi var. australe Parodi. Parodi, L.R. 1940. Estudio crítico de las gramíneas Austral- Americanas del género Agropyron. Rev. Mus. La Plata II, Bot. 12: 27-28, fig. 9, Lam. III.

Paratipo: Argentina: Tierra del Fuego, Río Grande. Leg. A. Castellanos. Fecha: 12/01/1933. BA 7611 .

Nota 1: un estudio posterior (Seberg, 1989), sinonimiza esta variedad con Elymus glaucescens Seberg.

Nota 2: en la descripción original figura el ejemplax como Castellanos n%611 (Buenmus), en lugar de Castellanos $s / n, B A 7611$.

Agrostis nana (Presl.) Kunth var. aristata Griseb. Grisebach, A. 1879. Symbolae ad Floram Argentinam. Abh. Königl. Ges. Wiss. Göttingen 24: 294.

Cotipo: Argentina: Catamarca, Yacutala. Leg. P. Lorentz. Fecha: 02/1872. BA 39199. 


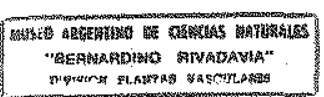

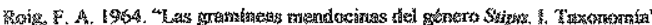

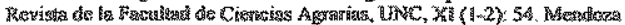

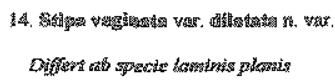

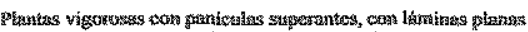

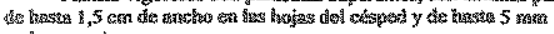
en las stiperioges

Material examinato:

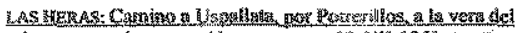

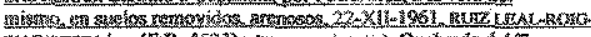

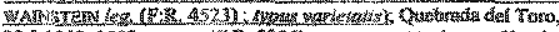

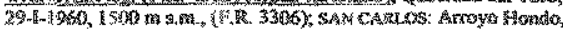

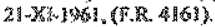

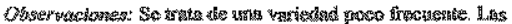

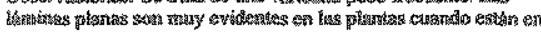

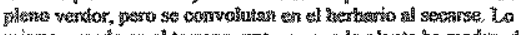

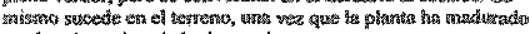

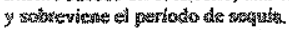

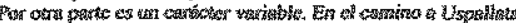

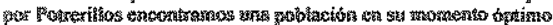

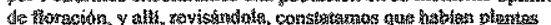

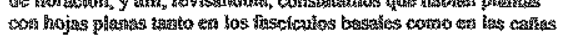

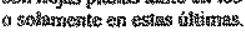

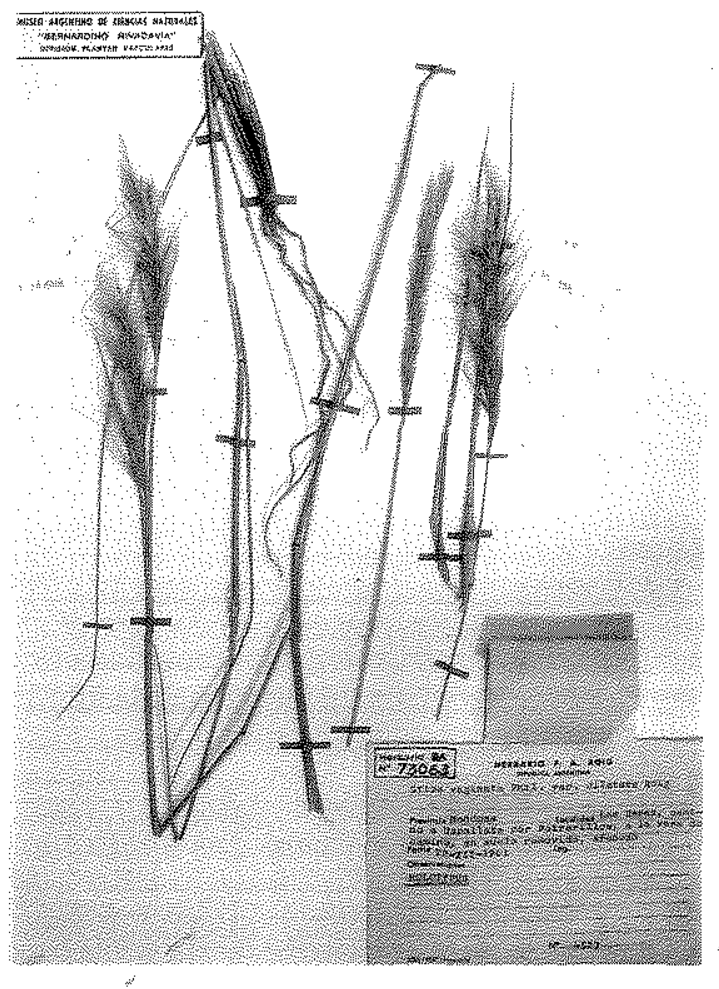

Fig. 1. Ejemplar tipo con su cita original y diagnosis correspondiente.

Nota 1: un estudio posterior (Rúgolo de Agrasar y Molina, 1992) sinonimiza esta variedad con Agrostis tolucensis Kunth.

Airopsis millegrana Griseb, Grisebach, C. 1874. Plantae Lorentzianae: 204.

Isotipo: Argentina: Tucumán, in monte Cuesta de Beico. Leg. P.G. Lorentz 772. Fecha: 1872. BA $38791 a$.

Nota 1: un estudio posterior, sinonimiza esta especie con Eragrostis airoides Nees.

Airopsis millegrana Griseb. Grisebach, C. 1874.

Plantae Lorentzianae: 204.

Isotipo: Argentina: Sierra de Tucumán.Leg. P.G. Lorentz 772. Fecha: 03/1872. BA 66186.

Nota: un estudio posterior, sinonimiza esta especie con Eragrostis airoides Nees.

Alopecurus aequalis Sobol. f. fluitans Parodi. Parodi, L.R. 1931. Revisión de las Gramíneas austroamericanas del géneroAlopecurus. Rev. Fac. Agron. Veter. 7 (2): 345-369.

Isotipo: Argentina: Tierra del Fuego, Río Grande. Leg. M. Doello Jurado 129. Fecha: 28/02/1921. BA 52909 .
Nota: un estudio posterior Mariano en Correa, N. (1978), sinonimiza este taxón con Alopecurus aequalis Sobol.

Alopecurus bonariensis Thell, et Parodi. Parodi, L.R. y A. Thellung. 1927. Alopecurus bonariensis Parodi et Thell. spec. nov. Fedde Repert. XXIII: 302-303.

Holotipo: Argentina: Prov, Buenos Aires, Avellaneda. Leg. L.R. Parodi 6526. Fecha: 11/10/ 1925. BA 1927/2.

Observación: habita praderas húmedas.

Aristida minutiflora Caro. Caro, J.A. 1961. Las especies de Aristida (Gramineae) del Centro de la República Argentina. Kurtziana 1: 151, fig. 5.

Paratipo: Argentina: Catamarea, Sierra del Alto, Vilismán. Leg. A. Castellanos. Fecha: 29/01/1944. BA 47646.

Aristida minutiflora Caro. Caro, J.A. 1961. Las especies de Aristida (Gramineae) del Centro de la República Argentina. Kurtziana 1: 151, fig. 5.

Paratipo: Argentina: Córdoba, Cordón Occidental, río Ojo de Agua. Leg. A. Castellanos. Fecha: 08/02/1944. BA 47657. 
Aristida pallens Cav, var. geminata Caro. Caro, J.A. 1961. Las especies de Aristida (Gramineae) del Centro de la República Argentina. Kurtziana 1: 171.

Paratipo: Argentina: San Luis, Sierras de San Luis, Canutal. Leg. A. Castellanos. Fecha: 21/12/ 1929. BA 29/253.

Aristida saycupersis Caro. Caro, J.A. 1961. Las especies de Aristida (Gramineae) del Centro de la República Argentina. Kurtziana 1: 160.

Holotipo: Argentina: San Luis, Laguna Sayape.Leg.

A. Castellanos. Fecha: 28/12/1925. BA 25/2732.

Aristida spegazzinit Arechav. Arechavaleta, J. 1895. Cuatro gramíneas nuevas y una conocida de la República Uruguaya. Anal. Mus. Nac. Buenos Aires 4: 177-179, fig. 1 .

Isotipo: Uruguay: "In campis graminosis, loco, ruta Independencia". Leg. J. Arechavaleta 1027. Fecha: 11/1893. BA. 38598.

Aristidla subulata Henrard. Henrad, J.T. 1928. A critical revision of the genus Aristida. Meded. Rijks. Herb. Leiden (54B); 612 614.

Isotipo: Argentina: Pampa central, Maracó. Leg.

A. Castellanos. Fecha: 08/01/1927. BA 27/117.

Atropis carinata Griseb. Grisebach, A. 1879. Symbolae ad Floram Argentinam. Abh. Königl. Ges. Wiss. Göttingen 24: 291.

Cotipo: Argentina: Jujuy, E1 Volcán. Leg. P. Lorentz y Hieronymus 741. Fecha: 05/1873. BA 39650.

Nota: Nicora (inéd.) in Zuloaga et al. 1994, sinonimiza esta especie con Diplachne uninervia (Pres1) L.R. Parodi.

Briza fusca Parodi. Parodi, L.R. 1922. Las gramíneas de la región de Concordia, (Prov. Entre Ríos). Rev. Fac. Agr. y Vet. Buenos Aires 4:93.

Clastotipo (fragmento de inflorescencia); Uruguay, Leg. J. Arechavaleta. Fecha: no consignada. BA 39910.

Nota: un estudio posterior (Nicora \& Rúgolo, 1981) sinonimiza esta especie con Briza lamarckiana Nees.

Briza paleapilifera Parodi. Parodi, L.R. 1920. Notas sobre las especies de Briza de la Flora Argentina. Rev. Fac. Agr. y Vet. 3: 124.

Sintipo: Argentina: Córdoba, próx. a la Capital. Leg. C. Lizer. Fecha: 1910. BA 39922.

Briza tandilensis Parodi. Parodi, L.R. 1920. Notas sobre las especies de Briza de la Flora Argentina. Rev. Fac. Agr. y Vet. 3: 132, fig. 5.

Clastotipo (fragmento de inflorescencia): Argentina: Prov. Buenos Aires, Sierras de Tandil. Leg. L.R. Parodi. Fecha: 01/11/1919. BA 39940.
Nota: un estudio posterior (Matthei, 1975) sinonimiza esta especie con Calotheca brizoides (Lam.) Desy.

Chloris castilloniana Lillo et Parodi. Parodi, L.R. 1918. Sobre las Chlorídeas de la República Argentina. Physis IV: 177-178, fig. 1-2.

Isotipo: Argentina: Tucumán, Amaicha, camino a Fuerte Quemado. Leg. L. Castillón 3450. (Herb. Lillo 16462). Fecha: 02/1914. BA 39452.

Observación: altitud $1800 \mathrm{~m}$ s.n.m.

Chloris castilloniana Lillo et Parodi var. pubescens Caro et Sánchez. Caro, J.A. y E.A. Sánchez. 1971. Chlorideae argentinas. Kurtziana VI: $230-231$.

Holotipo: Argentina: La Rioja, Los Llanos, Los Cerrillos. Leg. A. Castelianos. Fecha: 15/02/40. BA 33415.

Chloris dustichophylla Lag. var. argentino Hackel. Hackel, E. 1904. Contribución al conocimiento de las Gramináceas argentinas. Anal. Mus. Nac. Hist. Nat. Buenos Aires serie 3,4:113.

Isoparatipo: Argentina: Córdoba, prope Córdoba. Leg. T. Stuckert 11045. Fecha: 02/01/1902. BA 39432 .

Notá: un estudio posterior (Anderson, 1974) sinonimiza ešta variedad con Eustachys retusa (Lag.) Kunth.

Chloris grandiflora Ros. et Iz. Rosengurtt, B. y P. Izaguirre. 1968. Nuevas especies de Chloris de Uruguay y Paraguay. Bol. Soc. Arg. Bot. 12: 124m 128, fig. 3.

Paratipo: Argentina: Entre Rios, Concepción del Uruguay. Leg. R. Báez 340. Fecha: 11/1918. BA 39454 .

Nota: un estudio posterior (Anderson 1974) sinonimiza esta especie con Chloris canterai Arechav. var. grandiflora (Ros. et Iz.) Anderson.

Chloris parodiana Ros. et Iz. Rosengurtt, B. y P. Izaguirre 1968. Nuevas especies de Chloris de Uruguay y Paraguay. Bol. Soc. Arg. Bot. 12: 128-131, fig. 4.

Paratipo: Argentina: Chaco, Dpto. $1^{\circ}$ de Mayo, Colonia Benítez. Leg. A.G. Schulz 10997. Fecha: 25/02/1960. BA 73206.

Observación: habitat suelo alto arcilloso salitroso. Planta escasa, $25-40 \mathrm{~cm}$ alt.

Nota: un estudio posterior (Anderson, 1974) sinonimiza esta especie con Chloris canterai Arechav. var canterai.

Chloris parvispicula Caro et Sánchez. Caro, J.A. y E.A. Sánchez. 1971. Chlorideae argentinas. Kurtziana VI: 227-229, fig. 3.

Isotipo: Argentina: La Rioja, Los Llanos, Los Cerrillos. Leg. A. Castellanos. Fecha: 15/02/1940. BA 33416. 
Cheloris parvispicala Caro et Sánchez. Caro, J.A. y E.A. Sánchez 1971. Chlorideae argentinas. Kurtziana VI: $227-229$.

paratipo: Argentina: Catamarca, Sierra del Alto, Vilismán. Leg. A. Castellanos. Fecha: 29/01/1944. BA 47644 .

Chusquea ciliata Phil. Philippi, R. Linnaea XXXIII: 299.

Clastotipo (fragmento de inflorescencia): Chile: Algaroba. Leg. R. Philjppi (ex coll. Hosseus). F'echa: 1863. BA 24/1429.

Chersquea culeou Desv. f. longiramea Parodi. Parodi, L.R. 1941. Estudio sobre el género Chusquea en la Argentina. Rev. Arg. Agron. 8: 342343.

Isotipo: Argentina: Río Negro, Nahuel Huapí, Puerto Blest. Leg. A. Burkart 6348. Fecha: 06/ 02/1934. BA 12110.

Observación: nombre vulgar "colihue". Arbusto muy abundante en el bosque, raro en flor.

Nota: un estudio posterior (Nicora, 1978) sinonimiza esta forma con Chusquea culeou Desv.

Chusquea culeou Desv. f. longiramea Parodi. Parodi, L. R. 1941. Estudio sobre el género Chusquea en la Argentina. Rev. Arg. Agron. 8: 342 343.

Paratipo: Argentina: Neuquén, Brazo Bleste, arroyo Bravo. Leg. A. Castellanos. Fecha: 08/02/1938. BA 21867.

Nota: un estudio posterior (Nicora, 1978) sinonimiza esta forma con Chusquea culeou Desv.

Chusquea tenuiflora Phil. Philippi, R.A. $1859-$ 60. Plantarum novarum Chilensium. Linnaea XXX: 206-207.

Clastotipo (fragmento de inflorescencia): Chile: entre Cruces y San José. Leg. R. Philippi (Ex Coll. Hosseus). Fecha: 03/1858. BA 24/1428.

Cinnagrostis polygama Gris. Grisebach, A. 1874. Plantae Lorentzianae: 208-209.

Clastotipo: (rama de uno de los verticilos de la panícula): Argentina: Tucumán, in pratis $m$. Cuesta de Anfama. Leg. P.G. Lorentz. Fecha: 03/ 1872. BA 66142 .

Observación: gramínea robusta, hojas anchas.

Nota: un trabajo posterior (Parodi 1953) sinonimiza esta especie con Deyeuxia polygama (Gris.) Parodi.

Cynodon distichloides Caro et Sánchez. Caro, J.A. y E.A. Sánchez. 1969. Nuevas especies de Cynodon (Gramineae) de Uruguay y Africa. Kurtziana V: 254-258 fig. 1.

Paratipo: República Oriental del Uruguay. Depto. Maldonado: Punta Ballena. Leg. A. Castellanos. Fecha: 07/04/1941. BA 37036.
Cynodon grandispiculus Caro et Sánchez. Caro, J.A. y E.A. Sánchez. 1969. Nuevas especies de Cynodon (Gramineae) de Uruguay y Africa. Kurtziana V: 258-262.

Paratipo: República Oriental del Uruguay. Leg. M. Doello Jurado. Fecha: 02/1925. BA 25/294.

Cynodon grandispiculus Caro et Sánchez. Caro, J.A. y E.A. Sánchez. 1969. Nuevas especies de Cynodon (Gramineae) de Uruguay y Africa. Kurtziana V: 258-262.

Paratipo: República Oriental del Uruguay: Punta del Este, Depto: Maldonado. Leg. C. Osten 16278. Fecha: 15/03/1921. BA 24/1024.

Cynodon maritimus H.B.K. var. grandispiculus Caro et Sánchez. Caro, J.A. y E.A. Sánchez. 1969. Las especies de Cynodon. Kurtziana V: 234-236, fig. 2.

Isotipo: Argentina: Tucumán, Depto. Capital, Río Salí.Leg. S. Venturi 2737. Fecha: 31/01/1924. BA 24/227.

Observación: habitat orilla del río formando césped, altura s. n. m. 450 m.

Deschampsia cordilleranum Hauman. Haumañ, L. 1918. La végétation des hautés Cordillères de Mendoza. An. Soc. Cient. Arg, 86: 231-233.

Isotipo: Argentina: Mendoza, Las Cuevas, orilla del arroyo. Leg. L. Hauman. Fecha: 03/1918. BA 39306.

Digitaria fallens Parodi. Parodi, L.R. 1926. Dos nuevas especies de gramíneas de la Flora argentina. Physis VIII: $375-379$, fig. 2.

Paratipo: Argentina: Corrientes: Torrent. Leg. L.R. Parodi 5699. Fecha: 29/02/1924. BA 27/3.

Observaciones: planta de 40 a $60 \mathrm{~cm}$ abundante en el andén de la Estación Torrent.

Nota: un estudio posterior (Rúgolo, 1974) sinonimiza esta especie con Digitaria eriostachya $\mathrm{Mez}$.

Diplachne uninervia (Presl.) L.R. Parodi f. abbreviata Parodi. Parodi, L. R. 1927. Revisión de las gramíneas del género Diplachne. Rev. Fac. Agron. y Vet. VI: 15-19.

Isosintipo: Argentina: Jujuy, El Volcán. Leg. P. Lorentz y Hieronymus 741. Fecha: 05/1873. BA 39650.

Nota: Nicora (inéd.) in Zuloaga et al., 1994, sinonimiza esta forma con Diplachne uninervia (Presl.) Parodi.

Distichlis spicata (L.) Greene var, mexicana. Beetle. Beetle, A. 1943. The North American variations of Distichlis spicata. Bull. Torr. Bot. Club $70: 648$.

Isocotipo: Mexico: Durango city and vicinity. Leg. E. Palmer 182 bis. Fecha: 4 a 11/1896. BA 9283. 
Elymus agropyroides J. Presl. var. brevimucronatus Hauman. Hauman L. 1918. La végétation des hautes cordillères de Mendoza. An. Soc. Cient. Arg. 86: 233-234.

Lectotipo: Argentina: Mendoza, Puente del Inca, borde del río Mendoza. Leg. L. Hauman. Fecha: 01/1908. BA 55729a.

Nota: un estudio posterior (Seberg, 1998) sinonimiza esta variedad con Elymus angsulatus J. Presl.

Elymus erianthus Phil. var. spegazini (Kurtz) Hauman. Hauman L, 1917. Notes floristiques. An. Mus. Nac. Buenos Aires $29: 77$ y 410.

Sintipo: Argentina: Santa Cruz, Puerto Deseado, San Jorge. Leg. C. Ameghino. Fecha: 1900. BA 55734 .

Nota: un estudio posterior (Dubcovsky et al., 1997) sinonimiza esta especie con Leymus erianthus (Phil.) Dubcovsky.

Elymus erianthus Phil. var. spegazzini (Kurtz) Hauman. Hauman L. 1917. Notes floristiques. An. Mus. Nac. Buenos Aires 29: 77 y 410.

Sintipo: Argentina: Chubut, Trelew ${ }^{-T e c k a . ~ L e g . ~}$ N. Illín. Fecha: 12/1899. BA 55733.

Nota: un estudio posterior (Dubcovsky et al, , 1997) sinonimiza esta especie con Leymus erianthus (Phil.) Dubcovsky.

Erioneuron avenaceum (H.B.K.) Tateoka var. longiglume (Parodi) Anton. Anton, A.M. 1977. Notas críticas sobre gramíneas de Argentina, II. Kurtziana 10: 65-66.

Paratipo: Argentina: Tucumán, Tafí del Valle. Leg. A. Castellanos. Fecha: 27/01/1935. BA 14634.

Erioneuron pilosum (Buckley) Nash var. parodianum Sánchez. Sánchez, E.A. 1979. Anatomía foliar de las especies y variedades argentinas de los géneros Tridens y Erioneuron. Darwiniana 22(1-3): 171, fig. 3.

Holotipo: Argentina: La Pampa, Dpto: Lihuel Calel, Sierra de Lihuel Calel, en cerros. Leg. A. Krapovickas 3633. Fecha: 21/12/1947. BA 70615. Observación: sobre suelo pedregoso.

Erioneuron pilosum (Buckley) Nash var. parodianum Sánchez, Sánchez, E.A. 1979. Anatomía foliar de las especies y variedades argentinas de los géneros Tridens y Erioneuron. Darwiniana $22(1-3): 171$, fig. 3.

Topotipo: Argentina: La Pampa, Sierra de Lihuel Calel. Leg. O. Boelcke y E. Nicora 8131. Fecha: 30/11/1969. BA 79665.

Erioneuron pilosum (Buckley) Nash var. parodianum Sánchez, Sánchez, E.A. 1979.
Anatomía foliar de las especies y variedades ar gentinas de los géneros Tridens y Erioneuron. Darwiniana 22(1-3): 171, fig. 3 .

Paratipo: Argentína: La Pampa, Entre Gral. Acha y Lihuel Calel. Leg. A. Castellanos. Fecha: 23/ 11/1941. BA 37466.

Erioneuron pilosum (Buckley) Nash var. parodìanum Sánchez. Sánchez, E.A. 1979. Anatomía foliar de las especies y variedades argentinas de los géneros Tridens y Erioneuron. Darwiniana 22(1-3): 172, fig. 3 .

Topotipo: Argentina: La Pampa, Dpto. Lihuel Calel, Sierra de Lihuel Calel. Leg. E. Sánchez y M. Arriaga 1219. Fecha: 19/10/1979. BA 70706. Nota: se realizó a este ejemplar datación isotópica de carbono $(\delta \mathrm{C} 13 \%=-13,5)$.

Erionezron pilosum (Buckley) Nash var. porrodíarezum Sánchez. Sánchez, E.A. 1979. Anatomía foliar de las especies y variedades argentinas de los géneros Tridens y Erioneuron. Darwiniana 22(1-3): 172 , fig. 3 .

Isoparatipo: Argentina: La Pampa, Dpto. Lihuel Calel Sierra de Lihuel Calel. Leg. O. Boelcke y E. Nicora 8137. Fecha: 30/11/1969. BA 70637.

Erioneuron pilosum (Buckley) Nash var. parodianum Sánchez. Sánchez, E.A. 1979. Anatomía foliar de las especies y variedades argentinas de los géneros Tridens y Erioneuron. Darwiniana 22(1-3): 172, fig. 3 .

Isoparatipo: Argentina: La Pampa, Dpto. Lihuel Calel Ruta 152, sierra de Lihuel Calel, frente al A.C.A. Leg. O. Boelcke et al. (BAA 11560). Fecha: 06/11/72. BA 70636a.

Erioneuron pilosum (Buckley) Nash var. parodianum Sánchez. Sánchez, E.A. 1979. Anatomía foliar de las especies y variedades argentinas de los géneros Tridens y Erioneuron. Darwiniana 22(1-3): 172 , fig. 3 .

Isoparatipo: Argentina: Ruta 152, sierra de Lihuel Calel, frente al A.C.A. Leg. O. Boelcke et al. Fecha: 06/11/72. BA 70636.

Festuca cochabambana Alexeev. Alexeev, E.B. 1985. New taxa and typification of Festuca (Poaceae) of Bolivia. J. B. Soc. URSS 70 (9); 1241\% 1243, fig. 1-4.

Isotipo: Bolivia: Depto: Cochabamba, Prov. Chaparé, Incachaca. Leg. J. Steinbach 8976. Fecha: 29/01/1929. BA 30/2414.

Festuca distichovaginata Pilg. var. carazana (Pilg.) St.-Yves subvar. angustiflora St.Yves. Saint-Yves, A. 1927. Contribution a létude des Festuca de l'Amérique du Sud. Candollea 3: 307. 
Isotipo: Argentina: Salta, Sierra del Cajón, La Laguna. Leg. D. Rodríguez 1324. Fecha: 23/01/ 1914. BA 40222 .

Nata: un estudio posterior (Türpe, 1969) sinonimiza este taxón con Festuca distichovaginata Pilg.

\section{Hestued distichovaginata Pilg. var. cardand} (Pilg.) St.-Yves subvar. angustiflort St.Yves. Saint-Yves, A. 1927. Contribution a l'étude des Festuca de l'Amérique du Sud. Candollea 3:307. Isotipo: Argentina: Salta, Sierra del Cajón, La Laguna, Leg. D. Rodríguez 1324. Fecha: 10/02/ 1914. BA 40221.

Nota: un estudio posterior (Türpe, 1969) sinonimiza esta subvariedad con Festuca disticovaginata Pilg.

Festuea lilloi Hackel. Hackel E. 1914. Quatrième contribution a la connaissance des graminées argentines. Ann. du Conserv. et du Jard. bot. Genéve 17: 300 .

Isotipo: Argentina: Tucumán, Lara. Leg. D. Rodriguez 287. Fecha: 28/01/1912. BA 40200a.

Nota: un estudio posterior (Türpe, 1969) sinonimiza esta especie con Festuca tucumanica E.B. Alexeev.

Fi estuca Longidiuma Parodi. Parodi, L. R. 1953. Las especies de Festuca de la Patagonia. Rev. Arg. Agr. 20 (4): 214-216, fig. 5.

Holotipo: Argentina: Tierra del Fuego, Isla de los Estados, Puerto Cook. Leg. A. Castellanos. $\mathrm{Fe}$ cha: 04/01/1934. BA 12825.

Nota: un estudio posterior (Matthei, 1982) sinonimiza esta especie con Festuca cirrosa (Speg.) Parodi.

Festuca longidiurna Parodi. Parodi, L. R. 1953. Las especies de Festuca de la Patagonia Rev. Arg. Agr. 20 (4): 214-216, fig. 5.

Paratipo: Argentina: Tierra del Fuego, Isla de los Estados, Caleta Luisa. Leg. A. Castellanos. Fe. cha: 11/01/1934. BA 12865.

Nota: un estudio posterior (Matthei, 1982) sinonimiza esta especie con Festuca cirrosa (Speg.) Parodi,

Festuca longidiuma Parodi. Parodi, L. R. 1953. Las especies de Festuca de la Patagonia. Rev. Arg. Agr. 20 (4): 214-216.

Paratipo: Argentina: Tierra del Fuego, Isla de los Éstados, Puerto Crosley. Leg. A. Castellanos. Fecha: 04/02/1934. BA 12861.

Nota: un estudio posterior (Matthei, 1982) sinonimiza esta especie con Festuca cirrosa (Speg.) Parodi.

Festuca longidiurna Parodi. Parodi, L. R. 1953. Las especies de Festuca de la Patagonia. Rev. Arg. Agr. 20 (4): 214-216, fig. 5.

Paratipo: Argentina: Tierra del Fuego, Isla de los Estados, Puerto San Juan. Leg. A. Castellanos. Fecha: 06/01/1934. BA 12843.
Nota: un estudio posterior (Matthei, 1982) sinonimiza esta especie con Festuca cirrosa (Speg.) Parodi.

Gymnopogon glaber Caro. Caro, J.A. 1982. Las especies de Gymnopogon de la Flora Argentina. Dominguezia 5: 17-19. fig. 5 .

Holotipo: Argentina: Salta, Depto. Anta, Cerro Maldonado. Leg. A. Ragonese 131. Fecha: 06/ 1934. BA 13068.

Gymonopogon grandiflores Ros., Arr. et lzag. Rosengurtt, B. E., B.R. Arrillaga de Maffei y P. Izaguirre de Artucio. 1968. Especies nuevas y notas taxonómicas de gramíneas en Uruguay y $P_{c-}$ raguay. Bol. Fac. Agr. Montev. 103: 20-22.

Paratipo: Argentina: Buenos Aires, Saavedra, Sierras de Currumalán. Leg. L. Hauman. Fecha: 25/03/1918. BA 39538.

Gymmopogon gratediflorzs Ros., Arr. et Izag. Rosengurtt, B. E., B.R. Arrillaga de Maffei y P. Izaguirre de Artucio. 1968. Especies nuevas y notas taxonómicas de gramineas en Uruguay y $\mathrm{Pa}$ raguay. Bol. Fac. Agr. Montev. 103: 20-22.

Paratipo: Argentina: Buenos Aires, Sierras de Balcarce. Leg. A. Castellanos. Fecha: 04/1925. BA 25/1387.

Fierochloë gunchelï Parodi. Parodi, L. R. 1941. Revisión de las gramíneas sudamericanas del genero Hierochloë. Rev. Mus. La Plata, (n.s.) Bot. 3(14): 197. Paratipo: Chile: Valdivia. Leg. Otto Buchtien. Fecha: 07/11/1899. BA 27/20.

Hierochloë redolens (Vahl) Roem. et Schult. var. micrantha Parodi. Parodi, L. R. 1941. Revisión de las gramíneas sudamericanas del género Hierochloë. Rev. Mus. La Plata, (n.s.) Bot. 3(14): 197.

Paratipo: Chile: Provincia Magallanes, región Cameron. Leg. A. Donat 357. Fecha: 11/1930. BA 10862.

Hordeum bonariense Parodi et Nicora. Parodi, L.R. y E.G. Nicora. 1977. Novedades en el género Hordeum (Gramineae). Hickenia I (11): 55-62, fig. 1. Holotipo: Argentina: Buenos Aires, San Antonio de Areco, ea.»El Ombu», Leg. O. Boelcke 7835. Fecha: 29/10/1961. BA 70401.

Observación: común en campo bajo.

Nota: un estudio posterior (Bothmer et al., 1982) sinonimiza esta especie con Hodeum flexuosum Nees ex Steud.

Hordeum chilense Brongn, var. pseudosecalinum Hauman. Hauman, L. 1916. Hordeum spontanés de la Flore Argentine. An. Mus. Nac. Hist. Nat. Buenos Aires XXVIII: 270-273. 
Cotipo: Chile: Coihim, cerca de Puerto Montt. Leg. L. Hauman (misit Fuentes). Fecha: no determinada. BA 40455.

Nota: un trabajo posterior (Brothmer et al.,1980) sinonimiza esta variedad con Hordeum chilense Roem. et Schult.

Hordew jubatum L. var. pampeam Hauman. Hauman, 1. 1916. Hordeum spontanés de la Flore Argentine. An. Mus. Nac. Hist. Nat. Buenos Aires XXVIII: 284-286.

Isosintipo: Argentina: Buenos Aires, Chacabuco, Villafañe, estancia San Gregorio, Leg. No determinado (Herb. Min. Agr. 1742), Fecha: 03/1912. BA 40444.

Nota: un trabajo posterior (Parodi \& Nicora, 1978) sinonimiza esta variedad con Hordeum jubatum $\mathrm{L}$.

Hordewm maritimam With. subsp. gusso踝ean (Parlat.) Ascherson et Grachner var. palagonicum Hauman. Hauman, L. 1916. Hordeum spontanés de la Flore Argentine. An. Mus. Nac. Hist. Nat. Buenos Aires XXVIII: 287-288.

Isotipo: Argentina: Santa Cruz, Puerto Deseado. Leg. C.M. Hicken. Fecha: 13/01/1914. BA 40436 .

Nota: un trabajo posterior (Parodi \& Nicora 1978) sinonimiza esta variedad con Hordeum patagonicum (Hauman) Covas subsp. patagonicum.

Ichnanthus lilloi Hack. Hackel, E. 1887. Gramineae in A. Engler und K. Prantl. Die Natürlichen Pflanzenfamilien, Leipzig, 2(2): 32-39,

Isotipo: Argentina: Tucumán, La Criolla. Leg. D. Rodríguez 512. Fecha: 18/04/1912. BA 11278.

Nota: un trabajo posterior (Zuloaga, 1981) sinonimiza esta especie con Ichnanthus minarum (Nees) Döll.

Ichnanthus lilloi Hack. Hacke1, E. 1887. Gramineae in A. Engler und K. Prant1. Die Natürlichen Pflanzenfamilien, Leipzig, 2(2): 32-39. Isotipo: Argentina: Tucumán, La Criolla, quebradas. Leg. D. Rodríguez 512. Fecha: 04/1912. BA 11280.

Nota: un trabajo posterior (Zuloaga, 1981) sinonimiza esta especie con Ichnanthus minarum (Nees) Döll.

Koeleria grisebachii Domin. var. mendocinensis Hauman L. 1912 La végétation des hautes Cordillères de Mendoza. Bot. Lap. 3: 342 .

Isotipo: Argentina: Mendoza, Haute Cordillère, Laguna de Horcones. Leg. L. Hauman. Fecha: 03/ 1918. BA 39788.

Nota: un trabajo posterior (Nicora, 1978) sinonimiza esta variedad con Koeleria mendocinensis (Hauman) C.E. Calderón ex Nicora.
Laziola gracillima Prodoehl Prodoehl, A. 1922. Oryzeae monographice describuntur. Bot. Arch. 1: 241.

Isotipo: Mexico: Estado de Jalisco, shallow water near Guadalajara. Leg. C.G. Pringle. Fecha:02/ 10/1891. BA 9263.

Lycurus alopecturoides Griseb. Grisebach, A. 1874. Plantae Lorentzianae: 207-208.

Isotipo: Argentina: Catamarca, Belén. Leg. P.G. Lorentz 548. Fecha: 02/1872. BA 38492a.

Nota: un trabajo posterior (Sánchez E. \& Rúgolo de Agrasar, 1986) sinonimiza esta especie con Lycurus setosus (Nutt.) Reeder.

Melica andina Hauman. Hauman, L. 1917. Notes floristiques. An. Mus. Nac. Buenos Aires XXIX: $404-405$.

Paratipo: Argentina: Mendoza, Depto. Las Heras Precordillera de la Crucesita. Leg. E. Carette. Fecha: 12/1906. BA 39831.

Observación: este ejemplar también constituye el Isolectotipo de Melica andina Hauman var. trinervis Papp. (Papp, C. 1928. Monographie dex Südamerikanischen Arten der Gattung Melica L. Feddes Repert. Spes. Nou. Regni Veg. 25: 97-160).

Melica andina Hauman. Hauman, L. 1917. Notes floristiques, An. Mus. Nac. Buenos Aires XXIX: 404-405.

Neotipo: Argentina: Mendoza: valle del río Mendoza, Puente del Inca. Leg. L. Hauman. $\mathrm{Fe}$ cha: 01/1908. BA 39832.

Nota 1: Torres (1980), con el fin de restablecer el criterio de Hauman designa este ejemplar como Neotipo.

Nota 2: un trabajo posterior (Muñoz-Schick, 1983-1984) sinonimiza esta especie con Melica chilensis J. Presl.

Melica argyrea Hackel var. pilosa Papp. Papp, C. 1929. Monographie der südamerikanischen Arten der Gattung Melica L. Feddes Repert. Spec. Nov. Regni Veg. 25: 108.

Isotipo: Argentina: Córdoba, Río Cuarto. Leg. T. Stuckert. 15305. Fecha: 18/12/1905. BA 39840.

Nota: un estudio posterior (Torres, 1980) sinonimiza esta variedad con Melica argyrea Hack. var. argyrea.

Melica decumbens Papp. Papp, C. 1928. Monographie der südamerikanischen Arten der Gattung Melica L. Feddes Repert. Spec. Nov. Regni Veg. 25: 139.

Isotipo: Argentina: Tucumán, Lara. Leg. D. Rodríguez 325 (in herb. Stuckert 11180), Fecha: 13/02/1912. BA 65805 .

Nota: un trabajo posterior (Torres, 1980) sinonimiza esta especie con Melica lilloi Bech. 
Pancum enneanewrum Griseb. Grisebach, A. 1874. Plantae Lorentzianae: 213-214.

Isotipo: Argentina: Tucumán, cum praecedente pr. Siambón. Leg. P.G. Lorentz. Fecha: 1872. BA 38238.

Nota: un trabajo posterior (Zuloaga, 1975) sinonimiza esta especie con Panicum ovuliferum Trin.

Pandezu oblongatme Griseb. Grisebach A. 1874. Plantae Lorentzianae: 213.

Isotipo: Argentina: Tucumán, In umbrosis udis sylvarum subtropicarum pr. Siambón. Leg. P.G. Lorentz. Fecha: 1872. BA 38429.

Nota: un trabajo posterior (Türpe, 1973) sinonimniza esta especie con Setaria oblongata (Griseb.) Parodi.

Paspalume epilis Parodi. Parodi, L. R. 1926. Dos nuevas especies de gramíneas de la Flora Argentina. Physis (Buenos Aires) 8:372-374, fig 1 .

Holotipo:Argentina: Misiones, Posadas (Santa Inés). Leg. L.R. Parodi 5437. Fecha: 08/02/1924. BA 27/1.

Observación: planta perenne, densamente cespitosa. Habita bañados.

Nota: un trabajo posterior (Parodi, inéd.) sinonimiza esta especie con Paspalum ovale Nees ex Steud.

Paspatum hammanie Parodi. Parodi, L. R. 1925. Una nueva especie de Gramineas de la República Argentina. Com. Mus. Hist. Nat. Buenos Aires 2 (21): $217-219$, fig. 1 .

Isotipo: Argentina: Buenos Aires, Chañá Miní (Delta del río Paraná). Leg. L.R. Parodi 4930. Fecha: 14/01/1923. BA 11357.

Observación: la etiqueta de este ejemplar dice "typo de la especie?", la escritura es la del Ing. L.R. Parodi y con la misma escritura tacha la fecha y anota $1 / 1923$.

Paspalum Ratumanii Parodi. Parodi, L. R. 1925 Una nueva especie de Gramineas de la República Argentina. Com. Mus. Hist. Nat. Buenos Aires 2 (21): 217-219, fig. 1 .

Topotipo: Argentina: Buenos Aires, Delta del río Paraná. Leg. L. Hauman. Fecha: 12/1916. BA 11194.

Piptochaetium uruguense Griseb. Grisebach, A. 1879. Symbolae ad Floram Argentinam: 297

Isotipo: Argentina: Entre Ríos, Concepción del Uruguay. Leg. P. G. Lorentz 471. Fecha: 13/11/ 1875. BA 38961.

Poa acutifolia Hauman. Hauman, L. 1917. Notes floristiques. An. Mus. Nac. Hist. Nat, Buenos Aires XXIX: 405-407.

Holotipo: Argentina: Haute Cordillère de Mendoza (río Tupungato et río de Plomo vers
3000 mètres d'altitude). Leg. L. Hauman. Fecha: no consignada. BA 39988.

Poa atropidiformis Hackel. Hackel, E. In Dusén, P. 1900. Die gefässpfllanzen der Magellansländer. Svenska Exped. Magell. $3(5): 224$.

Topotipo: Argentina: Tierra del Fuego, Río Cullen. Leg. A. Castellanos. Fecha: 16/01/1933. BA 7585 .

Observación: L. R. Parodi (quien determinara este ejemplar) anota en la etiqueta "ejemplar valioso por provenir del hugar tipo".

Pod bergi Hieron. Hieronymus, G. 1879. Bol. Acad. Nac. Córdoba. IIl: 374-375.

Clastotipo (fragmento de inflorescencia): Argentina: Río Negro inférieur, Médanos del río Ne gro. Leg. C. Berg. Fecha: 10/1874. BA 40014.

Observación: en la etiqueta de este ejemplar dice: "fragmento del ejemplar origina".

Poa chilensis Trin. em. Desv, var. desvauxit Hauman. Hauman, L. 1918. La végétation des hautes Cordillères de Mendoza. An. Soc. Cient. Arg. 86:238.

Isotipo: Argentina: Mendoza, Cord. Tunuyán, Vallecito, $2700 \mathrm{~m}$. Leg. R. Sanzin. Herb. L. Hauman 32 var. Fecha: 01/1917. BA 40021.

Observación: ejemplar femenino.

Nota: un estudio posterior (Nicora, 1978) sinonimiza esta variedad con Poa denudata Steud.

Poc chubutensis Spegazzini. Spegazzini, C. 1902 Nova addenda ad Floram Patagonicam An. Mus. Nac. Hist. Nat. Buenos Aires VII: 196-198.

Clastotipo (fragmento de inflorescencia) y Fototipo: Argentina: Chubut, Tecka-Choique. Leg. N. Mlín. Fecha: (verano 1901). BA 40022.

Observación: en la etiqueta se consigna: "especimen auténtico ex herb. Spegazzini, en ded. L. Hauman".

Nota: un estudio posterior (Nicora, 1978) sinonimiza esta especie con Poa borchesii Phil.

Poa huecu Parodi. Parodi, L.R. 1950. Las gramineas tóxicas para el ganado en la Repúbllica Argentina. Rev. Arg. Agron. 17(3): 183.

Isoparatipo: Argentina: Netuquén, Cordillera del Viento. Cajón del Atreuco.Leg. A. Ragonese 284. Fecha: 25/01/1935. BA 14297.

Observación: ejemplar masculino.

Nota: existe preparado de polen en la División Paleobotánica N¹018, de 1977.

Poa huecu Parodi. Parodi, L.R. 1950. Las gramíneas tóxicas para el ganado en la Repúbllica Argentina. Rev. Arg. Agron. 17(3): 183. 
Isoparatipo: Argentina: Neuquén, Zapala. Leg. A. Ragonese 70. Fecha: 08/01/1935. BA 14319. Observación: ejemplar femenino.

Poa iridifolia Hauman. Hauman 1. 1917. Notes floristiques. An. Mus. Nac. Buenos Aires XXIX: 407408.

Holotipo: Argentina: Buenos Aires, Sierra de la Ventana, cerca del hotel S. de la Ventana. Leg. L. Hauman. Fecha: 11/1914. BA 40030.

Observación: ejemplar masculino.

Pac ragonesei Nicora. Nicora, E.G. 1995. Notas sobre los géneros Poa y Puccinellia (Gramineae). Hickenia II (33): 145-146 fig. 2.

Isotipo: Argentina: Salta, Depto. de Anta, Sierra Centinela. Leg. A. Ragonese 272. Fecha: 07/1934. BA 13059.

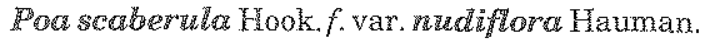
Hauman L. 1918. La végétation des hautes Cordillères de Mendoza. An. Soc. Cient. Arg. 86: 238. Holotipo: Argentina: Mendoza, Depto. Las Heras, Valle del río Mendoza, cerca de Puente del Inca. Leg. L. Hauman 34. Fecha: 01/1908. BA 40060. Nota: un estudio posterior (Nicora, 1978) sinonimiza esta variedad con Poa parviceps Hack.

Poa schizantha Parodi. Parodi, L. R. 1940. Una nueva especie de Poa de la Flora Argentina. Notas Mus. La Plata V, Bot. 31: 325 .

Isotipo: Argentina: Buenos Aires, Monte Hermoso. Leg. L.R. Parodi 13672. Fecha: 08/11/1940. BA 40061.

Observación 1: ejemplar femenino.

Observación 2: habita en las dunas de la costa atlántica.

Schizachyrium biciliatum Roseng., B, B. Arrill. et P. Izag. Rosengurtt, B., B. Arrillaga y P. Izaguirre. 1970. Gramíneas uruguayas: 195-199. Paratipo: República Oriental del Uruguay, Malabrigo. Leg. L. Hauman. Fecha: 11/1922, BA 11121.

Nota: un estudio posterior (Türpe, 1984) sinonimiza esta especie con Schizachyrium sanguineum (Retz.) Alston.

Nota 2: ejemplar mezclado con Schizachyrium hirtiflorum Nees.

Sporobolus maximus Hauman. Hauman L. 1921. Deux graminées géantes de la flore argentine. Physis V: 52-54.

Holotipo: Argentina: Salta, Cafayate, valle del río San Carlos. Leg. L. Hauman. Fecha: 11/1919. BA 39098.

Observación: terreno salado, con Distichlis, Nitrophila occidentalis, Juncus acutus, etc.
Stipa ameghino Speg. Spegazzini, C. 1901. Stipeae Platenses. Anal. Mus. Nac. Montevideo $4(2)$.

Argentina: Santa Cruz, Puerto Deseado. Leg. C. Ameghino. Fecha: 1899-1900. BA 71368.

Observación: En la tarjeta original el Ing. Parodi escribe: "comparado con el tipo guardado en el Instituto C. Spegazzini, La Plata. Tal vez sea de la colección original".

Nota: si bien este no constituye un ejemplar tipo, se trata de material histórico, por ello es incluido en este catálogo.

Stipa atacamensis Parodi. Parodi, L. R. 1960. Las especies del subgénero Pappostipa de la República Argentina. Rev. Arg. Agron. 27(3-4): 85-87.

Paratipo: Chile: Provincia de Coquimbo, cordillera, Baños del Toro, Río Malo. Leg. A. L. Cabrera 3517. echa: 18/01/1936. BA 20428.

Síipa bomand Hauman. Hauman L. 1917. Notes floristiques. An. Mus. Nac, Buenos Aires XXIX: 397 399.

Holotipo: Argentina: Jujuy, Susques. Leg. E. Boman. Fecha: 02/07/1903. BA 38664.

Observación: en un cañón profundo. Desarrolla ácido cianídrico. Nombre vulgar vizcachera hembra.

Nota: un trabajo posterior (Peñailillo, 1996) transffere esta especie como Anatherostipa bomani (Hauman) Peñailillo, el título lleva por fecha 1996, pero este volumen no fue realmente distribuido hasta agosto de 1997. A su vez (Torres, 1997) transfiere esta especie como Nicoraella bomanii (Hauman) Torres, efectivamente publicado y distribuido en marzo de 1997.

Stipa charruana Arechav. Arechavaleta, J. 1895. Las gramíneas uruguayas. Anal. Mus. Nac. Buenos Aires 4:181-183.

Isotipo: Uruguay: "in campo arcilloso". Leg. Coll. Prof. J. Arechavaleta 1029. Fecha: nov, et ded 1894?. BA 38673.

Nota: un trabajo posterior (Barkworth, 1990) transfiere este ejemplar como Nassella charruana (Arech.) Barkworth.

Stipa frigida Phil. var. parvispictula Parodi. Parodi, L. R. 1960. Las especies del subgénero Pappostipa de la República Argentina. Rev. Arg. Agron. 27(3-4): 91.

Paratipo: Argentina: San Juan, Depto. Iglesia, al $\mathrm{N}$ de Rodeo, mina Fierro Nuevo. Leg. V.R. Perrone. Fecha: 25/02/1950. BA 54901.

Stipa frigida Phil. var parvispicula Parodi. Parodi, L. R. 1960. Las especies del subgénero Pappostipa de la República Argentina. Rev. Arg. Agron. 27(3-4): 91. 
Paratipo: Argentina: San Juan, Depto. Iglesia, al $\mathrm{N}$ de Rodeo, mina Fierro Nuevo. Leg. V.R. Perrone. Fecha: 27/02/1950. BA 54904.

Observación: glumas $15 \mathrm{~mm}$, superan el genículo.

Shipa hieronymusid Pilg. Pilger, R. 1920. Gramineae austro-americanae imprimis Weberbawerianae V Engler's. Bot. Jahrb. 56(2): 123:24.

Fototipo: Argentina: La Rioja: Cuesta del Peñón, al S de la Laguna Brava. Leg. G. Hieronymus y G. Niederlein 314. Fecha: 26/02/1879. BA 80048.

Stipa Iatifolia Hack. et Arechav. Hackel, D y J. Arechavaleta. 1895. Las gramíneas uruguayas. Anal. Mus. Nac. Buenos Aires 4: 183, fig. 4.

Isotipo: Uruguay: «in. rupium ad margines...»Leg. Herb. Prof. J. Arechavaleta 1030. Fecha: 12/1894. BA 38802.

Nota: un estudio posterior (Cabrera \& Torres, 1970) sinonimiza esta especie con Stipa megapotamica Spreng. ex Trin.; actualmente transferida como Nassella megapotamica (Spreng. ex Trin.) Barkworth.

Stipa molfinoi Speg. Spegazzini, C. 1925. Stipae platenses novae vs. criticae. Rev. Arg. Bot. I: 3032.

lsotipo: Argentina: Misiones, San Javier-Apóstoles. Leg. J.F. Molfino. Fecha: 22/02/1922. BA $25 / 1540$.

Observación: en campo desmontado.

Nota: un trabajo posterior (Parodi, 1928) sinonimiza esta especie conStipa sellowiana Nees ex Trin. et Rupr.

Stipa pugionata Caro et Sánchez. Caro J.A. y E. A. Sánchez. 1973. Las especies de Stipa del subgénero Jarava. Kurtziana 7:109-112, fig. 9.

Holotipo: Argentina: Catamarca, Depto. Antofagasta, Incahuasi. Leg. Hueck 512. Fecha: 01/04/1950. BA 65817.

Stipa speciosa Trin. y Rupr. f. abscondita Roig. Roig, F. 1964. Las gramineas mendocinas del género Stipa, I Taxonomía. Rev. Fac. Cienc. Agr. XI(12): 36 .

Isotipo: Argentina: Mendoza, Las Heras, Cruz del Paramillo. Leg. Herb. F.A. Roig 4929. Fecha: 11/ 02/1964. BA 73064.

Stipa speciosa Trin. y Rupr. var. breviglumis Parodi. Parodi, L. R. 1960. Las especies del subgénero Pappostipa de la República Argentina. Rev. Arg. Agron. 27(3-4): 74-75.

Holotipo: Argentina: San Juan, Depto. Iglesias, al $\mathrm{N}$ de Rodeo, mina Fierro Nuevo, Leg. V.R. Perrone. Fecha: 27/02/1950. BA 549041/2. Observación: glumas de $16 \mathrm{~mm}$.

Stipa speciosa Trin. y Rupr. var. breviglumis Parodi. Parodi, L. R. 1960. Las especies del subgénero Pappostipa de la República Argentina. Rev. Arg. Agron. 27(3-4): 74-75.

Paratipo: Argentina: San Juan, Depto. Iglesia, al $\mathrm{N}$ de Rodeo, mina Fierro Nuevo. Leg. V.R. Perrone. Fecha: 24/02/1950. BA 54905.

Observación: glumas de $16 \mathrm{~mm}$.

Stipa vaginata Phil, f. contracta Roig. Roig, F. 1964. Las gramineas mendocinas del género Stipa,

I Taxonomía. Rev. Fac. Cienc. Agr. 11(1-2): 54.

Topotipo: Argentina: Mendoza, Depto. Las Heras, Paramillo de Uspallata. Leg. F.A. Roig 4920. Fe. cha: 11/02/1964. BA 72966.

Stipa vaginata Phil. var. dilatata. Roig. Roig, F. 1964. Las gramineas mendocinas del género Stipa, I Taxonomía. Rev, Fac. Cienc. Agx. XI(1-2): 54.

Holotipo: Argentina: Mendoza, Depto. Las Heras, camino a Uspallata por Potrerillos. Leg. Herb. F.A. Roig 4523. Fecha; 22/12/1961. BA 73063. Observación: a la vera del camino, en suelo removido, arenoso.

Stipa vaginata Phil. f. tnmersa Roig. Roig, F. 1964. Las gramíneas mendocinas del género Stipa, 1 Taxonomía. Rev. Fac. Cienc. Agr. XI(1-2): 54.

Cotipo: Argentina: Mendoza, Depto. Las Heras, cumbre del Paramillo de Uspallata. Leg. F.A. Roig 4308. Fecha: 22/01/1960. BA. 72948.

Stipa vaginata Phil. f. rigida Roig. Roig. F. 1964. Las gramíneas mendocinas del género Stipa, I Taxonomía. Rev. Fac. Cienc. Agr. XI(1-2): 54.

Topotipo: Argentina: Mendoza, Depto. Las Heras, Cruz del Paramillo, Leg. F.A. Roig 4921. Fecha: 11/02/19/64. BA 72956.

Tridens avenacea (H.B.K) Hitchc. var, longiglumis Parodi. Parodi, L.R. 1937. Revisión de las gramíneas del género Tridens de la Flora Argentina. Rev. Arg. Agr. 4 (4): 255.

Isotipo y Fototipo: Argentina: Catamarca, Andalgalá. Leg. L.R. Parodi (colección P. Jörgensen 1718). Fecha: 06/03/1916. BA 69660. Observación: flor con un solo estambre, antera de $0,75 \mathrm{~mm}$.

Nota: un trabajo posterior (Anton, 1977) sinonimiza esta especie con Erioneuron avenaceum (H.B.K.) Tateoka var. longiglume (Parodi) Anton.

\section{BIBLIOGRAFIA}

Anderson, D.E. 1974. Taxonomy of the genus Chloris (Gramineae). Brigham Young Univ. Sci. Bull. Biol. Ser. 19 (2): 1-133.

Anton. A.M. 1977. Notas críticas sobre gramíneas de Argentina II. Kurtziana 10: 51-67.

Barkworth. M.E. 1990. Nassella (Gramineae, Stipeae): 
revised interpretation and nomenclatural changes. Taxon 39 (4): 597-614.

Bothmer, R. von, N. Jacobsen, R.B. Jorgensen \& E.G. Nicora. 1982. Revision of the Hordeum pusillum group. Nord. J. Bot. 2 (4): 307-321.

Bothmer, R. von, N. Jacobsen \& E.G. Nicora. 1980. Revision of Hordeum sect. Anisolepis. Neushi Bot. Not. 133: 539-554.

Cabrera, A.l \& M.A. Torres. 1970. Stipa. En A. L. Cabrera. Flora de la Provincia de Buenos Aires. Colec. Cient. INTA $6(2): 1-624$.

Dubcovsky, J., A.R. Schlatter \& M. Echaide. 1997. Genome analysis of South American Elymus (Triticeae) and Leymus (Triticeae) species based on variation in repeated nucleotide sequences. Genome 40: 505-520.

Hauman, L. \& G. Vanderveken. 1917. Catalogue des Phanérogames de l'Argentine. An. Mus. Nac. Hist. Nat. Buenos Aires 29: 1-351.

López, M.E. 1988. Catálogo de los tipos depositados en el Herbario del Museo Argentino de Ciencias Nattzrales "Bernardino Rivadavia" (BA). I. Monocoliledóneas: Potamogetonaceae, Alismataceae, Cyperaceae, Xyridaceae, Eriocaulaceae. Rev. Mus. Argentino Cienc. Nat. "Bernardino Rivadavia", Botánica "7(1): 1 -8.

Löve, A. 1984. Conspectus of Triticeae, Feddes Repert $95(7-8): 425-521$.

Mariano, M. 1978. Alopecurus. En M.N. Correa, Flora Patagónica, Colecc. Cient. NTA 8 (3): 340-348.

Matthei, O.R. 1975. Der Briza-Komplex in Sudamerika: Briza, Calotheca, Chascolytrum, Poidium (Gramineae). Willdenowia 8: 1-168.

- 1982. El género Festuca (Poaceae) en Chile. Gayana Bot. 37: 1-62.

Muñoz-Schick, M. 1983-1984. Revisión de las especies del género Melica L. (Gramineae) en Chile. Bol. Mus. Nac. Hist. Nat. 40: 41-89.

Nicora, E.G. 1978. Gramineae. En M. N. Correa, Flora Patagónica. Colec. Cient. INTA 8 (3). 563 pp.

Nicora, E.G. \& Z.E. Rúgolo. 1981. Los generos sudamericanos afines a Briza L. (Gramineae). Darwiniana $23(1): 279-309$.

Parodi, L.R. 1927. Revisión de las gramíneas argentinas del género Diplachne. Reu. Fac. Agron. 6 (1): $21-43$.

- 1928. Notas sobre Gramíneas de la Flora Argentina. Physis (Buenos Aires) 9: 12-45.

- 1953. Gramíneas argentinas nuevas o críticas. II. Rev. Argent. Agron. 20 (1): $11-90$.
Inéd. Revisión de las especies argentinas del género Paspalum.

Parodi, L.R. \& E.G. Nicora. 1978. Hordeum. En M. N. Correa. Flora Patagónica. Colec. Cient. MNA. 8(3): $406-440$.

Peñailillo, B.P. 1996. Anatherostipa, un nuevo género de Poaceae (Stipeae). Gayana Bot. 53: 277-284.

Rúgolo de Agrasar, Z.E. 1974. Las especies del género Digitaria (Gramineae) de la Argentina. Darwintana 19:65-166.

Rúgolo de Agrasar, Z.E. \& A.M. Molina. 1992. Las especies del género Agrostis (Gramineae: Agrosteae) de la Argentina. Parodiana 7(1-2): 179-255.

Sánchez, E \& Z.E. Rúgolo de Agrasar. 1986. Estudio taxonómico sobre el género Lycurus (Gramineae) Parodiana 4 (2): 267-310.

Seberg, O. 1989. A biometrical analysis of the South American Elymus glcucescens complex (Poaceae: Triticeae). Pl. Syst. Etol. $166(1.2): 91-104$.

Seberg, O.\& G. Petersen. 1998. A taxonomic revision of the genus Elymus L. s.l. (Poaceae, Triticeae) in South America. Bot. Jahrb. Syst. 120 (4): 503-543.

Torres, M.A. 1980. Revisión de las especies argentinas del género Melica (Gramineae). Opera Lilloana 29: 1.115.

- 1997. Nicoraella (Gramineae) un nuevo género para América del Sur. Comisión de Investigaciones Científicas, Prov. Buenos Aires Monografía 13: 69-77.

Türpe, A,M. 1969. Las especies argentinas de Festuca (excluidas las patagónicas). Darwiniana $15(1-2)$ : 189-283.

- 1973. Novedades en Gramíneas para la Flora Argentina. Lilloa 33 (13): 283-292.

- 1984. Revision of the South American species of Schizachyrium (Gramineae).Kew Bull. 39 (1): 169178.

Zuloaga, F'.O. 1975. El género Panicum (Gramineae) en la provincia de Jujuy. Bol. Soc. Argent. Bot. 16 (12); $53-65$.

- 1981. Las especies argentinas del génerolchnanthus (Gramineae).Darwiniana 23 (1): 189-221.

Zuloaga, F.O., E.G. Nicora, Z.E. Rúgolo de Agrasar, O. Morrone, J. Pensiero \& A.M. Cialdella. 1994. Catálogo de la familia Poaceae en la República Argentina. Missouri Bot. Gard. 47, 178 pgs. 